FORMATION Formation emploi

Revue française de sciences sociales

106 | avril-juin 2009

Pêle-mêle

\title{
La portée formatrice de l'expérience : le cas des employeurs du secteur des services
}

Die Bildungsfunktion der Erfahrung: Arbeitgeber im Diensleistungssektor

The formative impact of experience: employers in the services sector

El alcance formador de la experiencia : los empleados del sector servicios

Franck Bailly, Alexandre Léné et Marie-Hélène Toutin

\section{(2) OpenEdition}

Journals

Édition électronique

URL : http://journals.openedition.org/formationemploi/1927

DOI : 10.4000/formationemploi.1927

ISSN : 2107-0946

Éditeur

La Documentation française

Édition imprimée

Date de publication : 1 juin 2009

Pagination : 41-58

ISSN : 0759-6340

Référence électronique

Franck Bailly, Alexandre Léné et Marie-Hélène Toutin, « La portée formatrice de l'expérience : le cas des employeurs du secteur des services », Formation emploi [En ligne], 106 | avril-juin 2009, mis en ligne le 01 juin 2011, consulté le 10 décembre 2020. URL : http://journals.openedition.org/ formationemploi/1927 ; DOI : https://doi.org/10.4000/formationemploi.1927

(c) Tous droits réservés 


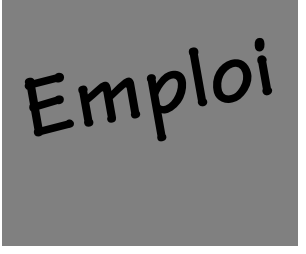

\title{
La portée formatrice de l'expérience : entretiens auprès d'employeurs du secteur des services'
}

Par Franck Bailly, Alexandre Léné et Marie-Hélène Toutin*

\begin{abstract}
Les périodes de la scolarité passées en entreprises occupent une place importante dans les dispositifs de formation professionnelle. Pourtant, les stages n'ont une portée formatrice qu'à certaines conditions. Le lien établi entre expérience, temps de présence en entreprise et connaissance est au final tout relatif.
\end{abstract}

\begin{abstract}
Aujourd'hui, quasiment tous les acteurs sociaux prônent un rapprochement entre le système scolaire et le monde du travail. La coopération écoleentreprise serait une caution d'efficacité de la formation professionnelle. L'Alternance, qui désigne un mode de formation associant des séquences de formation académique et des périodes en entreprise serait, de ce point de vue, une sorte d'idéal pédagogique. Un tel rapprochement se justifierait par le fait que les périodes en entreprise, sous forme de stage ou d'apprentissage, auraient, par la connaissance du monde du travail qu'elles procurent, une portée formatrice. Elles permettraient de rendre opérationnels et de donner un sens à des savoirs scolaires (Malglaive, 1996; Merle, 1994) ou d'acquérir des connaissances par la pratique (Vergnaud, 1991). L'expérience professionnelle et la formation scolaire
\end{abstract}

\footnotetext{
${ }^{1}$ Sans engager leur responsabilité, nous tenons à remercier nos collègues Y. Bensaâdoune, E. Chatel et C. Guegnard ainsi que trois rapporteurs anonymes pour leurs remarques et commentaires d'une version précédente de ce texte.
}

* Franck Bailly est maître de conférences en économie à l'université de Rouen. II mène ses recherches au sein du CARE (Centre d'analyse et de recherche en économie) en économie de l'éducation et en économie du travail. Récemment, il a publié : "The role of employers' beliefs in the evaluation of educational output", en 2008, dans The journal of Socio-Economics et "La construction de la qualité de L'éducation. L'exemple des bacs professionnels tertiaires », avec E. Chatel, dans L'économie des conventions : méthodes et débats, (sous la direction de Eymard-Duvernay F.) à La Découverte.

Alexandre Léné est maître de conférences en économie des ressources humaines à l'université de Lille 1. II enseigne à Telecom-lille 1 et mène ses recherches au sein du CLERSE |Centre Lillois d'études et de recherches sociologiques et économiques). Ses travaux portent sur la formation, l'insertion des jeunes, la gestion des compétences. II a publié récemment: "Rémunérer les compétences: I'entreprise peut-elle tenir ses promesses ? », en 2008, dans la Revue Française de Gestion, et « Détournements de main-d'œuvre et externalités de la formation dans un modèle de concurrence imparfaite », en 2005, dans Économie Appliquée. 
Marie-Hélène Toutin est chargée d'études au centre régional associé du Céreq de Lille. Elle est membre du CLERSE et travaille sur les questions de formation et d'apprentissage. Elle a publié avec B. Cart : "Correspondance entre formation et emploi: I'exemple des sortants de filière professionnelle de niveau bac", Des formations pour quels emplois? (sous la direction de J.F. Giret), en 2005.

constitueraient deux moyens, en partie complémentaires et substituables, d'acquérir des connaissances (Combes, 1996 ; Mallet et Vernières, 1981).

Cette portée formatrice des expériences de travail pourrait expliquer, selon Bonnal, Fleury et Rochard (1999) et Bonnal, Clément et Mendes (2005) la meilleure insertion professionnelle des élèves qui ont suivi la voie de l'apprentissage. Les auteurs comparent la situation des lycéens professionnels à celle des apprentis (pour les mêmes spécialités de formation et de diplômes préparés) sept mois après l'achèvement de leur formation. Leurs résultats montrent que l'insertion des jeunes formés par apprentissage est effectivement meilleure si on compare leur taux de chômage à celui des anciens élèves des lycées professionnels ou la nature des emplois auxquels ils accèdent, qui sont plus fréquemment des emplois sous contrat «ordinaire» (contrat à durée déterminée). L'influence positive des premières expériences professionnelles sur l'insertion ne concerne d'ailleurs pas seulement celles acquises en cours de scolarité. Par exemple, Balsan, Hanchane et Werquin (1996) montrent que l'expérience professionnelle des jeunes en début de vie active conditionne également fortement leur accès à des emplois stables ou, tout au moins, la nature des emplois trouvés après la phase de primo-transition. Ainsi, l'expérience professionnelle resterait un élément recherché par les entreprises (Béduwé, Espinasse et Tahar, 2000)² malgré l'émergence, en France, à partir des années 80, d'un nouveau modèle de gestion de l'emploi marqué par une accentuation de l'usage du diplôme comme indicateur d'un niveau de compétence générale (Verdier, 2001).

\footnotetext{
2 Notons que les résultats des travaux étrangers corroborent ceux des analyses françaises. $C f$. par exemple, Light (2001); Molitor et Leigh (2005).
}

Malgré ces éléments, toute période passée en entreprise peut-elle être considérée comme formatrice? Une part importante de la littérature économique répond de façon affirmative, comme si naturellement l'expérience était formatrice. D'autres travaux sont plus mesurés. Ils relient l'expérience professionnelle à l'activité de travail et à son caractère éprouvant. L'adoption de ce point de vue retire au temps passé en entreprise son caractère systémiquement producteur d'apprentissages et transforme la question initiale qui devient : qu'est-ce qui rend l'expérience formatrice ? À cet égard, l'objectif de notre contribution est double. Il est, d'abord, de donner un éclairage factuel aux éléments de réponses apportés par les analyses théoriques. Ensuite, de montrer, sur la base du matériau empirique recueilli, que ce qui compte dans la production de l'expérience a aussi à voir avec la représentation que l'on se fait de la portée formatrice du travail, représentation qui n'est pas indépendante des tensions employeur-employé qui caractérisent la relation salariale.

La première section de notre article rappelle la manière dont est traitée la question de l'expérience professionnelle dans la littérature économique. Elle indique également les choix méthodologiques qui découlent du cadre théorique retenu. Les deux sections suivantes présentent les éléments qui ressortent de l'exploitation du matériau empirique. De façon plus précise, la deuxième section indique les différentes dimensions sur lesquelles l'individu est mis à l'épreuve et la nature de l'expérience produite. Dans la troisième section sont analysés les supports qui comptent dans la production d'expérience. Enfin, dans la quatrième section, nous dépassons les points de vue exprimés dans le matériau empirique pour en souligner les implications quant à la place de l'entreprise dans le système de formation professionnelle. Cette analyse critique de la portée formatrice de l'expérience professionnelle nous amènera finalement à nuancer le consensus en la matière.

\section{FAIRE EXPÉRIENCE DU TRAVAIL : ÉLÉMENTS DE PROBLÉMATISATION}

La littérature économique relative à l'expérience professionnelle est abondante. Nous ne chercherons 
ici qu'à en délimiter les contours. Pour nombre de travaux économiques, le temps passé en entreprise est synonyme, presque mécaniquement, d'expérience professionnelle et d'apprentissages. En conséquence, la question de la production de l'expérience et de sa portée formatrice est évacuée... puisque l'expérience est formatrice. Une telle position tient sans doute, en partie, au fait que ces travaux n'abordent pas l'expérience par le biais du travail. Pour saisir cette question et en comprendre les enjeux, il faut en effet recentrer le propos sur l'activité de travail. Plusieurs analyses retiennent cette perspective et insistent sur le fait que le travail met à l'épreuve les personnes en les confrontant à des situations qu'ils doivent dépasser.

\section{L'expérience professionnelle comme boîte noire}

L'expérience professionnelle occupe, dans les travaux des économistes, une place singulière. Depuis les analyses pionnières de Mincer (1958), de nombreux travaux empiriques ont souligné son influence (Lemelin, 1998). Désormais, il s'agit d'une variable classique pour l'économiste qui analyse le marché du travail, variable qui pourtant a pour elle-même peu suscité de réflexion de la part des économistes. Son processus de production, c'est-à-dire l'analyse des éléments significatifs dans la production de l'expérience professionnelle, semble avoir peu retenu l'attention. L'expérience est généralement assimilée à du temps passé dans l'entreprise, à de l'ancienneté, qui naturellement produit des connaissances.

Cette assimilation nous semble caractéristique de la théorie du capital humain (Becker, 1964 ; Mincer 1958), mais aussi d'autres contributions majeures. C'est par exemple le cas de l'analyse d'Arrow (1962) lorsqu'il considère que l'apprentissage est «le produit de l'expérience» (p. 155) et qu'il choisit comme indicateur d'expérience les investissements cumulés, au motif que chaque nouvelle machine change l'environnement de la production et suscite ainsi de nouveaux apprentissages. De même, Rosen (1972b) note que l'hypothèse fondamentale est que les individus apprennent de leur expérience de travail, et fait dépendre les connaissances des quantités produites. Rosen (1972a) ainsi que Blinder et Weiss (1976) précisent que les firmes diffèrent dans leurs capacités à produire de l'expérience. Elles offrent des opportunités variables de se former et d'accumuler du capital humain, bien que l'origine de ces différences ne soit pas approfondie. Les explications d'un tel positionnement à l'égard de l'expérience professionnelle sont sans doute multiples. D'une part, l'analyse du processus de production de l'expérience, et d'une façon plus générale du capital humain, n'est pas toujours l'objet de ces travaux. Plus que ses origines, ils cherchent à comprendre les conséquences de l'expérience professionnelle. D'autre part, lorsque cette analyse est menée, elle reste marquée par une vision mécanique des phénomènes qui s'explique par l'influence, encore très présente à l'époque, de la fonction de production néoclassique. Enfin, dans ces travaux, l'expérience est analysée à travers le produit et plus rarement par le biais du travail, lequel, lorsqu'il est évoqué, renvoie là aussi, en partie à cause d'un effet d'époque, à une vision taylorienne.

\section{Le travail comme activité qui éprouve}

Pour une part importante, l'économie du travail marque une rupture au tournant des années 70 (Favereau, 1986). Les économistes entrent dans la boîte noire de l'entreprise. Celle-ci est désormais représentée comme un système d'incitation et de contrats entre les différents protagonistes. On peut alors comprendre que, depuis, l'analyse de la production et notamment la production d'expérience semble moins les préoccuper. Certains travaux continuent néanmoins de s'y intéresser, bien qu'ils aient des objectifs et s'inscrivent dans des courants de pensées différents. Ils abordent la question de l'expérience et de sa portée formatrice en faisant plus explicitement référence que ne le font les analyses précédentes à l'activité de travail. Celle-ci est envisagée, d'une façon ou d'une autre, comme une activité à laquelle les personnes sont confrontées, qui donc les éprouve c'est-à-dire qui est vécue par eux comme une épreuve et qui peut susciter de leur part engagement, apprentissage et expérience. Si la catégorisation des analyses est une opération délicate, plusieurs d'entre-elles semblent toutefois retenir cette dernière perspective.

C'est par exemple le cas de Stiglitz (1987), qui met l'accent sur la diversité des activités et souligne que 
cela se traduit par des phénomènes de complémentarités et d'externalités entre les différents apprentissages $^{3}$. Flueckiger (1976) met en avant le fait que l'individu doit être confronté à une série de stimuli provenant de son environnement pour enclencher un processus d'apprentissage, c'est-à-dire qu'il doit être confronté à des situations nouvelles. Levy-Garboua (1994) a une vision assez proche lorsqu'il assimile l'apprentissage sur le tas à un processus de découverte par lequel l'individu réalise successivement des expériences. De même, Foray (2001, p. 40) note que le processus d'apprentissage « est constitué en fait d'une suite d'expériences non planifiées. Ces expériences sont elles-mêmes provoquées par l'apparition de problèmes non anticipés au moment de la conception, ou bien de problèmes anticipés mais non résolus».

Grasser (1996) et Chatel (2007) nous semblent aller encore un peu plus loin dans cette direction. Grasser s'appuie sur les travaux de March et Simon (1958) et d'Argyris et Schon (1978). Il montre que les processus d'apprentissage individuels et organisationnels sont effectivement générés par l'activité de travail elle-même lorsque les individus sont confrontés à des situations problématiques qui les amènent à restructurer les règles et les schémas de pensée en vigueur dans l'organisation. Dans la lignée de la philosophie pragmatiste de Dewey, Chatel lie l'expérience au fait d'agir lorsque l'action ne va pas de soi, c'est-à-dire que les habitudes, les idées ou les connaissances des individus ne réussissent pas à conduire cette action à son terme. Pour continuer, ils doivent les mettre en question et les faire évoluer. Pour qu'il y ait expérience, il faut donc qu'il y ait trouble et dépassement du trouble. Mais l'expérience professionnelle peut aussi être reliée à l'activité de travail en un autre sens. Par analogie avec l'opposition classique qui structure l'économie de l'éducation entre théorie du capital humain et théorie du signal, Vincens (2001) fait en effet valoir que le caractère éprouvant du travail peut, parallèlement à la production d'apprentissages, être un moyen de révéler à l'individu lui-même et à l'employeur ses qualités.

Une certaine proximité existe entre cette manière dont les analyses économiques appréhendent la question de

\footnotetext{
${ }^{3}$ Par exemple, les apprentissages réalisés à un moment donné dans un domaine particulier favorisent les apprentissages ultérieurs, dans le même domaine ou même dans d'autres domaines.
}

la production d'expérience professionnelle et sa portée formatrice et la façon dont elle est traitée dans d'autres disciplines. Par exemple, pour la psychologie cognitive, l'acquisition de connaissances résulte principalement d'un apprentissage par l'action en situation de résolution de problèmes. C'est en utilisant des connaissances déjà acquises et en les confrontant à des situations nouvelles et variées que ces connaissances s'améliorent et que de nouvelles s'acquièrent (Malglaive, 1996). «Une connaissance doit s'inscrire dans les contextes situationnels, interactionnels et référentiels de l'action pour être utilisée » (Toupin, 1991, p. 309). Cette approche propose donc une conception "active» des apprentissages réalisés en situation de travail. Les salariés ne sont plus simplement considérés comme des «contenants» dans lesquels on "déverse » des connaissances ou des savoirs (Guile et Griffiths, 2001). Ils se saisissent des différentes ressources présentes dans l'environnement de travail pour développer leurs compétences.

Rentrer dans l'expérience professionnelle par le biais de l'activité de travail, comme le font ces différents travaux, revient à faire perdre à l'expérience son caractère naturellement formateur. Celle-ci n'est plus le simple effet mécanique du temps passé à faire quelque chose mais un processus qui mobilise la personne dans une activité qui l'éprouve. Cette évolution de la représentation de l'expérience professionnelle, relativement à ce qu'elle était dans les premiers travaux cités précédemment, n'est sans doute pas indépendante de l'évolution du travail lui-même. Ce dernier se limite de moins en moins à la réalisation d'un produit ou d'un service à travers des tâches connues et rationalisées. Il devient une activité qui laisse des marges de liberté, qui mobilise les initiatives, la créativité dans un contexte marqué par l'incertitude (Stankiewicz, 2002). Mais cette évolution pose aussi la question des éléments qui vont compter dans la production d'expérience. Avant d'y répondre, nous préciserons les choix méthodologiques qui découlent de ce changement de perspective.

\section{Mise à l'épreuve, évaluation et choix méthodologiques}

Centrer l'analyse sur le caractère éprouvant du travail revient à reconnaître que ce dernier déstabilise les personnes ou les remet, en partie, en cause, c'est- 
à-dire qu'il questionne l'évaluation qui est faite de leur qualité, de leur environnement et de son influence. En suivant l'économie des conventions, et d'une façon plus générale ce que Orléan (2002) nomme le «tournant cognitif» en économie, nous ne considérerons pas que ce travail d'évaluation relève d'un simple processus d'objectivation d'une réalité cachée. L'évaluation est aussi l'expression d'un jugement de valeur exprimé par l'évaluateur en fonction de la représentation qu'il se fait de ce qui compte. Il existe ainsi une diversité de façons d'évaluer et de qualifier le travailleur, de définir ce qu'est un «bon travailleur». Le jugement s'inscrit donc dans un registre plus large où d'autres évaluations sont possibles, avec ce que cela peu comporter de conflictualité. Plusieurs travaux ont déjà montré le caractère fructueux de cette approche pour l'analyse du travail, notamment des pratiques de recrutement des entreprises (Dubernet, 1996; Eymard-Duvernay et Marchal, 1997 ; Salognon, 2006). Nous la retiendrons pour traiter de la question de l'expérience professionnelle. La mise à l'épreuve du travail des jeunes constitue en effet un moment décisif au cours duquel sont émis des jugements de valeur sur ces débutants.

D'un point de vue méthodologique, cette perspective invite à la réalisation d'entretiens qui apparaît comme un outil privilégié pour saisir et analyser les jugements et ce qui les fonde (Blinder, 1990; Bewley, 2002). Ces entretiens doivent alors être compris comme l'expression d'une évaluation particulière qui peut ne pas correspondre à celle d'autres catégories d'acteurs (ou du chercheur). Nous avons fait le choix de réaliser des entretiens auprès d'employeurs du secteur des services ( $c f$. encadré 1). Leur point de vue nous semblait particulièrement pertinent dans la mesure où ils occupent les positions hiérarchiques les plus élevées dans l'entreprise. Les décisions de gestion qu'ils seront amenés à prendre sur la base de leur évaluation auront donc une force particulière. De plus, le fait que les entretiens concernent les premiers contacts des jeunes débutants avec le monde du travail a incité les employeurs interrogés à expliciter leurs jugements et à les rendre plus intelligibles.

Les éléments qui ressortent de l'exploitation des entretiens sont présentés dans les deux sections qui suivent. S'ils fournissent un contenu empirique à certains arguments pointés par des travaux théoriques, ils soulignent également combien l'évaluation réalisée par les employeurs de la portée formatrice des périodes passées en entreprise est influencée par la représentation qu'ils se font de l'activité de travail. C'est en effet sur la base de leurs croyances et de leurs représentations que les agents prennent leurs décisions et agissent, comme l'avait notamment souligné en partie Spence (1973). Nous avons donc cherché à dégager les différents points de vue et à comprendre leur construction. Nous verrons ainsi que ces représentations ne sont pas indépendantes de la dimension conflictuelle qui caractérise la relation de travail et des questions de partage de la valeur qui s'y jouent.

\section{LE CARACTÈRE ÉPROUVANT DU TRAVAIL ET SES DIMENSIONS}

La période passée en entreprise et la mise au travail revêtent, pour le lycéen ou l'apprenti, un caractère éprouvant. Ils l'évoquent fréquemment et les employeurs le reconnaissent (Bailly, Chatel, Léné, 2008). À l'analyse des entretiens, cette dimension éprouvante et la nature de l'expérience qu'elle produit apparaissent étroitement liées aux qualités qui doivent, selon les employeurs, être mobilisées dans l'activité de travail. Mais elles sont aussi dépendantes de leur croyance ou de la représentation qu'ils se font du caractère «apprenable » ou non de ces qualités. Des entretiens, trois types de qualités ressortent. Le premier type concerne la dimension technique du travail. Le deuxième porte sur sa dimension sociale, c'est-à-dire sur la capacité à intégrer un collectif de travail et à en saisir les normes et valeurs. Ces deux premiers types de qualités sont considérés par l'ensemble des employeurs comme pouvant s'apprendre. Le dernier ensemble de qualités porte sur la dimension relationnelle et comportementale de la personne au travail. Au contraire des précédentes, ces qualités sont perçues par certains employeurs comme ne pouvant pas ou peu s'apprendre. L'épreuve du travail ne ferait ainsi que révéler des qualités considérées par les employeurs comme inscrites dans les personnes. 


\section{Encadré 1}

\section{Méthodologie}

Les résultats exploités ici proviennent d'une réponse à l'Appel à Projet de recherche Action Concertée $d^{\prime}$ Initiative «Éducation et formation : contextes et effet ». Ce travail vise à évaluer dans quelle mesure les périodes de stage (pour les formations scolaires) ou d'apprentissage (pour les formations en alternance) permettent de faire expérience. Sont étudiées les formations des baccalauréats et BTS (brevet de technicien supérieur) tertiaires des spécialités " commerce ", " hôtellerie-restauration 》 et "comptabilité ». Le choix de spécialités tertiaires se comprend, en premier lieu, par l'importance des activités de services dans l'emploi total et le gisement d'emplois qu'elles représentent (Gadrey, 2003), en second lieu, par le faible intérêt qui leur a été accordé, au contraire des formations industrielles plus fréquemment étudiées.

Nous avons utilisé des entretiens réalisés auprès de salariés et d'employeurs de la région Parisienne, de la Haute-Normandie et du Nord-pas-de-Calais. Nous présentons ici les résultats de l'exploitation des 25 entretiens réalisés auprès des employeurs. Les entreprises contactées ont été tirées de façon aléatoire dans les fichiers Siret de l'Insee. Ces entretiens semi-directifs se sont déroulés sur le lieu de travail de la personne interrogée sur les années 2005-2006-2007. D'une durée d'une heure en moyenne, ils ont porté sur trois thèmes principaux : (i) l'emploi exercé par le jeune accueilli en stage ou en apprentissage et les qualités qu'il requiert : (ii) le sens donné par les employeurs à l'expérience et les avantages qu'elle procure selon eux; (iii) les raisons pour lesquelles les employeurs accueillent les jeunes des formations enquêtées et les avantages et inconvénients qu'ils trouvent à la formule du stage ou de l'apprentissage. De façon complémentaire, nous avons également utilisé certains des 21 entretiens réalisés pour une précédente recherche (Bailly, 2004).

Les personnes interrogées occupent des positions hiérarchiques (expert-comptable, chef de rayon, responsable des ressources humaines, ou directeur d'hôtels-restaurants) qui leur confèrent un rôle de premier plan dans le choix et l'accompagnement des stagiaires ou des apprentis et, le cas échéant, de leur recrutement. Ces personnes sont majoritairement des hommes. Dans la grande distribution, les interviewés ont un niveau bac, complété parfois par un cursus dans une école de commerce spécialisée dans la distribution ou un BTS. Une seule personne interrogée possède un CAP (certificat d'aptitude professionnelle). Dans l'hôtellerie-restauration, la diversité des parcours est plus importante : certains interviewés ont un CAP, alors que d'autres possèdent un BTS spécialisé dans ce secteur, une maîtrise d'anglais ou un diplôme d'ingénieur. Enfin, dans le secteur de l'expertise comptable, les interlocuteurs avaient tous suivi des études supérieures (de niveau au moins égal au BTS et diplômes spécialisés) ; I'un d'entre eux avait le niveau bac et avait complété sa longue expérience professionnelle par de la formation continue.

\section{La dimension technique et socialisatrice du travail et l'apprentissage des qualités}

L'épreuve concerne d'abord la maitrise technique du travail. Cette première dimension est assez immédiate. Elle se retrouve dans les trois secteurs étudiés. Il s'agit pour le jeune de maîtriser le plan et les opérations comptables, les règles juridiques, les logiciels spécialisés, de connaître la mise en rayon des produits, la tenue des cadenciers, le fonctionnement des réserves, de savoir s'adresser au client, d'enre- gistrer les réservations, les encaissements, le traitement des objections et remboursements ou bien encore de connaître la disposition des couverts, les associations de mets et de vins.

Selon les employeurs des trois secteurs, ces connaissances ne peuvent s'acquérir que sur le terrain. Elles constituent les bases du métier qui, par leurs composantes spécifiques, viennent compléter les savoirs scolaires : "à l'école c'est de la base, c'est vraiment la base et une fois qu'ils sont en entreprise c'est là qu'ils apprennent plus de choses en fait, c'est sur le 
terrain qu'ils apprennent plus de choses parce qu'à l'école on voit bien le programme c'est vraiment globalisé (...)» (Mme CB, directrice, hôtellerierestauration). Ce rôle de complément se justifie par des raisons techniques. Par exemple, il s'explique par la différence entre les logiciels utilisés à l'école et ceux qui le sont dans les entreprises ou par l'évolution rapide de la réglementation qui ne permet pas une mise à jour en temps réel des programmes scolaires. Il se justifie également par l'irréalisme de l'enseignement lui-même qui, par nature, est en dehors de la « vraie » vie au travail. «Le stagiaire ne fait pas la relation entre l'enregistrement des travaux à l'école et la matérialité des pièces»; "Un avoir, un relevé de banque, ça se prend dans quel sens? $C$ 'est la réalité d'un bac pro, c'est la réalité d'un $B T S$, c'est la réalité d'un DECF (diplôme d'études comptables et financières), c'est la réalité de tout étudiant qui n'a pas vu de véritable document d'entreprise » (Mme CX, expert-comptable). De ce point de vue, certains employeurs, notamment dans l'hôtellerie-restauration, regrettent que les enseignants des lycées n'aient pas exercé ou n'exercent plus le métier qu'ils enseignent.

Mais les connaissances dont sont porteuses les périodes en entreprises ne se limitent pas à la seule dimension technique du travail. Les entretiens soulignent en effet l'importance de la dimension socialisatrice. Celle-ci s'exprime, dans les trois secteurs, par l'apprentissage du respect de la hiérarchie et des règles de fonctionnement de la vie collective et des horaires, notamment lorsqu'ils sont atypiques comme dans la grande distribution et l'hôtellerie-restauration: "Et puis, plus de rigueur, plus de règles en commun; c'est ça qui leur paraît peut-être plus dur par rapport à l'école. Je ne dis pas qu'il n'y a pas de règles à l'école mais nous vis-à-vis $d u$ client on est obligé d'imposer plein de choses, la tenue vestimentaire, l'hygiène, la propreté, bien sûr les horaires vis-à-vis des collègues quand ils sont en retard, je ne peux pas me le permettre puisqu'on ouvre le magasin à des heures précises et puis si on le permet à une personne c'est la foire d'empoigne... » (M. PJ, directeur, grande distribution) Le fait d'être placé en situation de travail permet donc au jeune de mieux comprendre le monde de l'entreprise, sa façon de fonctionner et d'intérioriser sa culture (ses objec- tifs, ses valeurs, son langage...) et cette intégration est le point d'aboutissement d'un processus qui se construit et s'apprend dans l'entreprise.

\section{La dimension relationnelle et subjective du travail et la révélation des qualités}

Dans la grande distribution et l'hôtellerie-restauration, comme dans d'autres activités de services, la figure $\mathrm{du}$ client occupe une place centrale (Ughetto et alii, 2002). Les exigences des employeurs relatives à des qualités subjectives (personnalité, comportement) en sont une expression. Agulhon (2003) pointe ainsi qu'aucune des petites annonces des professionnels de l'hôtellerie-restauration n'est exempte d'injonctions concernant les capacités relationnelles, l'enthousiasme ou la convivialité. Ces éléments se retrouvent également dans nos entretiens : "Les autres qualités, ce sont plus des qualités comportementales, des qualités qu'on a naturellement, c'est évidemment : la politesse, la ponctualité, être serviable. Dans notre métier, une qualité essentielle c'est d'être, d'aller audevant de ce que peut désirer la personne. Donc c'est quand on voit un fumeur en face de nous, lui tendre un cendrier, c'est quand on voit une corbeille vide sur une table, remettre $d u$ pain avant qu'on demande, sentir dans le regard d'un client le mécontentement ou l'attente, quelqu'un qui est en attente de quelque chose café, ou même l'addition, tout ça c'est du ressenti. Il faut avoir une attitude de service, c'est tout. » (M. AF, directeur, restauration)

Contrairement aux qualités techniques et de socialisation précédemment évoquées, les qualités relationnelles sont perçues par les employeurs de ces secteurs comme étant peu susceptibles de s'apprendre. Il y a certes une composante technique (liée notamment aux techniques de communication), mais ces qualités relationnelles dépendent avant tout, selon eux, de caractéristiques que les personnes possèdent en elles. En ce sens, elles relèvent d'une logique de naturalisation ou d'incorporation, qui les place en dehors de toute forme d'apprentissage, comme l'ont également pointé d'autres travaux (Bellier, 1998; Gadrey, Jany-Catrice, Pernod-Lemattre, 2003). Ces qualités sont analysées par les employeurs en termes de «sensibilité », de «tempérament», de « on est 
fait pour ça » ou de "fibre ». "Aussi c'est évident il faut une fibre. Je pense qu'on a ça en soi. Il y a des gens qui sont très charismatiques et le commerce c'est ça ils sont à l'écoute, des fois ce n'est pas évident et puis voilà c'est naturel. (...) Ensuite évidemment il y a l'apparence, y'a l'élocution, il y a... la propreté mais bon tout ça ce sont ensuite des qualités naturelles. Quelqu'un qui n'a pas tout ça à la base de toute façon ne fera pas ce métier... 》 (Mme P.P, directrice adjointe, hôtellerierestauration).

L'épreuve de la mise au travail est alors explicitement vue comme un moyen de révéler ces qualités "Et là l'intérêt (de la période en entreprise), c'est que pendant ces deux ans, ils voient ce que c'est, ils savent si ça leur plait. Il faut, on est fait pour ça ou on n'est pas fait pour ça (...) Il y a, on sent déjà les gens, les stagiaires qui ont cette sensibilité. (...) le stage permet au jeune de mesurer son intérêt pour le métier et de voir s'il a ou non la fibre commerciale » (Mme MP, responsable des ressources humaines, grande distribution). Dans la comptabilité, la situation est différente. D'une part, les qualités relationnelles sont moins présentes, au moins au niveau des postes qu'occupent les lycéens et apprentis des formations que nous avons étudiées. D'autre part, ces qualités sont considérées comme pouvant s'apprendre: "on a besoin d'un certain sens commercial, ça c'est vrai que c'est pas inné, et ça l'est d'autant moins sur les gens qui se dirigent vers la comptabilité, nous sommes de très mauvais vendeurs. Donc quelqu'un qui est bon vendeur en compatibilité c'est excessivement rare, tout simplement parce qu'il faudrait purement et simplement une formation de commercial, que l'on n'a pas à la base dans toutes les formations comptables." (M. LN, expert-comptable)

\section{SURMONTER L'ÉPREUVE DU TRAVAIL GRÂCE AUX SUPPORTS DE L'EXPÉRIENCE}

Les éléments précédents relativisent l'idée d'une expérience nécessairement formatrice. Ils corroborent, de ce point de vue, la distinction opérée par
Vincens (2001) entre l'expérience-acquisition et l'expérience-révélation. L'expérience peut en effet être vue par les employeurs comme la révélation de qualités qu'ils considèrent être déjà là. Mais au-delà, la littérature souligne aussi toute la complexité du processus, non systématique, qui va du travail aux apprentissages. La production d'expérience est en effet influencée à la fois par les caractéristiques de la personne et le contexte productif (Grasser et Rose, 2000). Outre la volonté de la personne et son engagement, l'aptitude à se former sur un poste de travail n'est en effet pas indépendante de la formation du travailleur. En raison d'effets de complémentarité, on peut penser que l'expérience est d'autant plus aisée à acquérir que la formation initiale a été élevée et que les capacités d'apprentissage de la personne sont importantes.

À cela s'ajoute le fait que le contenu et le mode d'acquisition de l'expérience professionnelle varient selon les situations de travail. Toutes les configurations productives ne sont pas équivalentes du point de vue des apprentissages qu'elles suscitent. Le potentiel didactique des situations de travail varie d'une entreprise à l'autre (Ashton, 2004). Koike (2002) a ainsi comparé le processus de formation des compétences dans différents types d'organisation. Il montre que l'organisation du travail peut favoriser la mise en rapport des différents savoirs lorsque les activités sont intégrées. La répartition et le chevauchement des responsabilités entre les membres de l'équipe facilitent la détection, la correction et l'utilisation pédagogique des erreurs. La production d'expérience peut ainsi être intensifiée par le travail en équipe où la coopération est la règle. Par opposition, un environnement de travail fractionné et cloisonné limite la circulation de l'information et réduit les interactions et les rétroactions. Il est donc de la responsabilité des organisations qui accueillent des débutants de penser à la façon dont l'environnement de travail peut faciliter les apprentissages (Guile et Griffiths, 2001). La pratique doit être structurée et accompagnée. Le « guidage de l'activité » ou «l'exposition accompagnée à la variété » apparaissent comme des éléments particulièrement importants (Savoyant, 1996). Cela est vrai pour l'acquisition des compétences «techniques» mais également pour la transmission des normes et des 
valeurs de l'organisation qui peut être formalisée ou institutionnalisée (Baker et Feldman, 1990) ${ }^{4}$.

Nous retrouvons une partie des éléments pointés par ces analyses dans les entretiens réalisés auprès des employeurs. Ces derniers mettent en avant le fait qu'il faut organiser l'activité de travail et que le passage de l'épreuve est influencé par la formation initiale reçue. Les employeurs insistent également sur l'implication du lycéen ou de l'apprenti. Mais le regard que portent les employeurs sur ces différents supports de la production d'expérience laisse transparaître les tensions qui traversent la relation de travail. En particulier, l'accent mis sur l'implication du jeune débutant tend à faire perdre de vue aux employeurs leur propre responsabilité.

\section{Organiser les activités pour surmonter l'épreuve}

Les employeurs interrogés reconnaissent et revendiquent leur responsabilité dans la transmission des connaissances techniques et des valeurs du monde de l'entreprise. La mise en forme des tâches et des activités pour le jeune accueilli s'inscrit dans cette perspective. Cette «didactisation» de la situation de travail est très présente dans l'hôtellerie-restauration. On guide, on accompagne, on encadre le lycéen ou l'apprenti pour l'amener progressivement au travail tel qu'il devrait être fait: "Faire les simulations et les jeux de rôles... je suis la cliente... là aussi ils sont au fait, c'est-à-dire qu'on leur apprend le poste d'accueil de l'hôtel: se présenter à tout le monde, accueillir des clients, faire les encaissements» (Mme PP, directrice adjointe, hôtellerie-restauration); «j'en ai consacré des samedis après-midi d'hiver à les former et tout ça. (...) puis après c'est en nous, c'est en étant là avec nous » (Mme $\mathrm{CB}$, directrice hôtellerie-restauration). Dans la grande distribution, la situation est assez similaire.

Mais pour les employeurs de ces deux secteurs, la nécessité de «didactiser» le travail s'arrête là où

\footnotetext{
${ }^{4}$ Il faut noter que la capacité inhérente de l'organisation à motiver les individus affecte également l'efficacité de l'apprentissage. Dans certaines organisations, plus que dans d'autres, les individus sont encouragés à apprendre : quand les connaissances sont visiblement utiles dans l'activité de travail et que les individus ont des opportunités de se rendre compte de la valeur, en termes économiques, de ce qu'ils apprennent.
}

commence leur croyance dans le caractère naturel des qualités. Puisqu'une partie du travail est considéré par eux comme prenant appui sur une fibre déjà là, il n'est pas organisé dans une logique d'apprentissage. "Je veux dire ça ne peut pas s'apprendre quoi, faut l'avoir au fond de soi » (M. RT, directeur, grande distribution). La réalisation correcte du travail vient alors en quelque sorte naturellement... en même temps qu'elle révèle ces qualités : "Et puis la fibre ou on aime faire un beau rayon, un beau facing et mettre des étiquettes comme il faut, là déjà on sent qu'il y a quelque chose qui va aller vers le client. » (Mme RP, responsable formation, grande distribution) Pour les experts-comptables, la croyance en l'apprentissage des qualités modèle l'ensemble de la profession (Bailly, 2008). Mais de façon paradoxale, la nécessité d'une organisation didactique de l'activité de travail est peu explicite dans leurs entretiens. Outre la structuration et l'organisation du travail et de l'activité comptable qui, de façon évidente, facilitent les apprentissages, cela tient au fait que toute situation de travail est vécue par les expertscomptables comme une situation d'apprentissage, source possible d'évolution des qualités des personnes: "Ce n'est pas parce qu'il (le bachelier en stage) avait des faiblesses qu'elles étaient définitives. Vous savez moi j'ai coutume de dire que l'expérience c'est la somme des erreurs qu'on a pu faire. » (M. C, expert-comptable)

Au-delà de l'organisation didactique de l'activité de travail, d'autres conditions doivent être remplies pour que l'activité soit formatrice ou révélatrice. Les employeurs des trois secteurs soulignent l'importance de la variété des situations rencontrées. Ainsi, dans la grande distribution: "si on n'a pas vécu au moins un Noël et un Paques et je dirais, une saison aussi d'été (...), on n'a pas une formation complète je dirais, de la saisonnalité, qui est quand même hyper importante » (M., LG, directeur, grande distribution) Dans l'hôtellerie-restauration, cette variété s'exprime à travers la saisonnalité, comme dans la grande distribution, mais aussi par la diversité des établissements fréquentés et leur qualité. Dans la comptabilité, les périodes en entreprises sont également jugées trop courtes par les employeurs. Elles ne permettent pas de suivre les dossiers dans le temps et de comprendre la logique comptable qui les organise. 
Enfin, les employeurs insistent, particulièrement dans la grande distribution et l'hôtellerie-restauration, sur le fait que les situations vécues en stages doivent se rapprocher le plus possible des situations auxquelles les jeunes seront confrontés dans leur activité professionnelle, une fois recrutés. C'est à ce titre que les employeurs de l'hôtellerie-restauration portent un regard critique sur la législation qui encadre les conditions de travail des apprentis ou stagiaires: «Non parce que pendant les stages, ils travaillent $d u$ lundi au samedi. Le dimanche ils n'ont pas le droit de travailler, les stages vous ne travaillez pas, vous travaillez sur les jours ouvrables, donc ça ne peut pas leur permettre de se rendre compte. Tout ce qui est jours fériés, ils ne travaillent pas. Quand ils viennent en stage juillet et août, le quatorze juillet et le quinze août ils ne travaillent pas. » (Mme CB, directrice, hôtellerie-restauration)

\section{L'influence de la formation initiale}

Pour certains employeurs, la formation initiale permet de faciliter le dépassement de l'épreuve que représente le travail. Son influence se retrouve à plusieurs niveaux. D'abord, la formation initiale permet de mieux organiser le travail et signale une capacité d'adaptation. Cela confère également une capacité à prendre de la distance et permet une vision plus large des problèmes. Par exemple, dans l'hôtellerie, les titulaires d'un diplôme de niveau BTS pourront être privilégiés : "Les BTS ont, globalement, on va dire, une meilleure ouverture d'esprit, globalement, après les personnalités restent. Mais deux ou trois années d'études, ne serait-ce qu'au niveau des différentes matières, au niveau de la réflexion, ça joue. (...) Quelqu'un qui fait des études, il va s'adapter n'importe où. » (M. PS, directeur, hôtellerie) Ce sont également des personnes qui sont supposées être capables de conduire de façon autonome leurs apprentissages, en allant rechercher ellesmêmes les informations ou les ressources nécessaires. Cela apparaît a contrario lorsque ce manque d'autonomie est dénoncé par les employeurs: «Je pense que dès le départ il y a un manque de culture au sens large du terme, c'est-à-dire de capacité à chercher dans les bouquins, à essayer de trouver l'information quelque part, à se poser des questions, (...) c'est-à-dire de l'incapacité à un instant donné d'utiliser les moyens dont on dispose tant en ressources externes qu'en ressources internes à la limite pour solutionner un problème, d'une façon générale.» (M. $\mathrm{BC}$, expert-comptable) Enfin, une formation plus longue confère une base, un socle de connaissances qui leur permet d'apprendre plus vite par la suite.

Toutefois, ce type de position ne se retrouve pas chez l'ensemble des employeurs. Le plus souvent, il s'agit de responsables qui, eux-mêmes, ont fait des études supérieures (type BTS par exemple) et qui rationalisent leur propre parcours a posteriori. Il s'agit également d'entreprises mettant en œuvre une politique de recrutement et de gestion des carrières spécifique. Ainsi, dans les chaînes hôtelières, les postes à responsabilité (gérant d'hôtel) sont attribués exclusivement à des jeunes ayant suivi ce type de formation. On peut sans doute voir là les effets de la hausse générale du niveau de diplôme sur le discours des employeurs, notamment des plus jeunes générations. Malgré l'importance de la formation initiale, l'implication et la motivation individuelle restent, selon les employeurs, deux des critères essentiels pour dépasser les épreuves. Cette caractéristique est très présente dans le commerce ou l'hôtellerierestauration, mais également dans la comptabilité.

\section{L'implication au travail}

En plus des éléments précédents, les employeurs insistent en effet fortement sur la nécessité, pour le jeune, de s'impliquer afin de surmonter l'épreuve dans ses différentes dimensions. Pour l'employeur, cette implication s'entend comme un dépassement qui consiste à aller au-delà de ce qui est demandé explicitement. Ainsi, par exemple, il ne s'agit pas seulement pour le jeune de vouloir apprendre, il doit vouloir en apprendre plus: "il était bouffeur de formation, il restait l'après-midi sans arrêt pour voir des choses, il était passionné de décoration de... il était très bon en salle, je lui ai donné tout ce que je pouvais lui donner à ce jeune là et il a fait des choses qu'il aurait pas dî faire dans le terme de son contrat d'apprentissage (...) (M. AF, directeur, restauration). Cela doit même amener le jeune à une certaine abnégation qui consiste à faire corps avec l'entreprise : "Qu'ils se fondent complètement dans l'entreprise c'est-à-dire qu'ils n'aient pas d'exigences, $n i$ 
d'horaires, ni de forces enfin je veux dire qu'ils soient capables d'accepter tout ce qu'on leur demande de faire, qu'ils disent pas "Oh bah ça non ça je ne veux pas faire” c'est surtout ça.» (M. LB, directeur, hôtellerie)

L'accent mis par les employeurs sur la nécessaire autonomie du jeune est une autre facette de cette exigence d'implication. Les entreprises attendent en effet de plus en plus de leur personnel non seulement qu'il réalise des activités déterminées selon des moyens définis, mais qu'il mette en œuvre sa capacité d'adaptation aux changements et qu'il mobilise son potentiel d'initiative (Stankiewicz, 2002), ce qui se traduit par des exigences accrues en termes d'autonomie ou de responsabilité. C'est notamment le cas des salariés débutants qui sont très souvent renvoyés à eux-mêmes. Il leur est explicitement demandé d'être acteur de leur formation, d'exprimer leurs besoins, voire de construire leur parcours. Lorsque le jeune ne joue pas ce rôle, il est considéré comme n'étant pas suffisamment motivé. Au contraire, lorsqu'il pose trop de questions, sollicite trop son tuteur ou n'apprend pas assez vite, il lui est reproché de ne pas être assez mature, assez autonome, comme cette jeune fille considérée comme pas assez adulte ou trop étudiante : "Moi honnêtement j'ai énormément de mal à lui faire comprendre qu'elle est adulte, qu'elle est employée chez $X$. et qu'elle me doit des comptes et qu'elle doit être là. Je trouve qu'ils restent trop gamins quoi (...) Bah... ça n'arrive pas motivé déjà, ça n'arrive pas très motivé et c'est très dépendant. Là, on finit la première année et honnêtement avec elle je n'ai pas encore pu aborder du tout ce qui est gestion. On n'y est pas, on n'a pas du tout attaqué ça, on en est encore à la marchandise. Et je vois là ...elle est revenue ce matin, elle est encore en train de me demander des choses qu'on a déjà abordées. Pas adulte, pas adulte. Bon c'est peut-être la personne qui est comme ça.» (M. XL, responsable textile, grande distribution) Cette « sur-implication » devient en ellemême une qualité attendue au même titre que celles relatives à la maîtrise technique du travail, à sa dimension socialisatrice ou relationnelle et comme cette dernière, elle est souvent considérée comme naturelle par les employeurs. Cette «vertu» serait une caractéristique propre de la personne, un élément de sa personnalité.
Dans ce contexte, le rôle de signal que jouent, au moment du recrutement, les premières expériences professionnelles ne s'inscrit pas (ou pas seulement) dans une logique de transférabilité d'un savoir-faire ou de connaissances acquises ailleurs. Il permet aussi de déceler chez les jeunes la capacité à s'impliquer, à accepter des conditions de travail difficiles ou pénibles. "Quand on reçoit les curriculum vitae, on regarde ce qu'ils ont déjà effectué comme stage. C'est la pénibilité du stage, si vous voulez, qui nous permet de voir si le jeune c'est quelqu'un qui en veut, ou qui en veut pas. Je dirais même quelqu'un qui n'a pas fait un stage dans la distribution mais qui va avoir choisi une entreprise où on sait que le travail, là-bas, ce n'est pas quelque chose de facile, ça peut être un travail à la chaîne, vous voyez ce genre de choses, celui qui va avoir fait cette démarche en intégrant le monde du travail, nous convient parfaitement. (...) Le fait d'avoir travaillé en usine eh bien c'est du travail posté, ça veut dire que la personne elle est prête à travailler à n'importe quelle heure, voyez-vous? C'est ça en fait et pour nous c'est le fait d'accepter certaines contraintes, si vous voulez, qui nous permet nous de nous dire celui-là c'est quelqu'un qui en veut, c'est quelqu'un qui a compris que dans le travail il faut se donner.» (Mme. L, responsable des ressources humaines, grande distribution) L'expérience est donc une mise à l'épreuve d'où seuls les jeunes les plus vaillants et qui font preuve d'une réelle abnégation émergeront.

Ces exigences de "sur-implication » exprimées par les employeurs ont une contrepartie. Ces derniers tendent en effet à faire porter sur le seul jeune la responsabilité de l'issue de l'épreuve. Si le lycéen ou l'apprenti surmonte l'épreuve, c'est qu'il a su faire preuve de vaillance et si échec il y a, la responsabilité lui en incombe également. Mais dans tous les cas, les manquements des employeurs sont rarement évoqués dans les entretiens. Lorsqu'ils le sont, ils font référence aux comportements... d'autres employeurs : « sinon il y a certains patrons qui abusent toujours. On voit bien des stagiaires qui sont malheureux. Moi j'en ai récupéré un une année, un été, qui était franchement exploité pendant deux semaines. Il était carrément exploité. On nous l'a renvoyé ici pour sauver la face. » (Mme $\mathrm{CB}$, directrice, hôtellerierestauration) 


\section{LES ENJEUX} DE LA PRODUCTION D'EXPÉRIENCE POUR LA FORMATION DES JEUNES

Nous avons ainsi tenté de caractériser, à partir de l'exploitation d'entretiens réalisés auprès d'employeurs, ce qui compte, selon ces derniers, dans la production de l'expérience professionnelle. On a notamment souligné la place des croyances ou des représentations des employeurs à l'égard du caractère apprenable ou non des qualités et l'accent qu'ils mettent sur l'implication des jeunes. Au-delà des conséquences théoriques - mieux définir le concept d'expérience et sa «fonction de production »- ces éléments, parce qu'ils ont des influences sur les pratiques de gestion effectives des entreprises, montrent combien le déplacement de la formation vers l'entreprise peut être problématique pour la formation professionnelle (Agulhon, 2002). Trois points permettront de l'illustrer. Le premier questionne la capacité de l'entreprise à être un lieu de formation relativement aux tensions qui la traversent. Le second concerne la reconnaissance professionnelle des qualités qui en découle. Enfin, le troisième porte sur la place de l'entreprise dans le système de formation.

\section{Les contradictions de l'entreprise comme lieu de formation}

Les employeurs du commerce et de l'hôtellerierestauration considèrent qu'une partie des qualités au travail ne s'apprend pas. Elles seraient incorporées en quelque sorte dans les personnes. On l'a signalé. Or si ces qualités sont considérées comme pouvant s'apprendre, l'employeur est plus enclin à endosser le rôle de formateur que si, de manière explicite ou non, il privilégie l'idée de naturalisation de ces qualités. L'entreprise se trouve alors interrogée comme lieu de formation: n'est-il pas paradoxal, d'un côté, de mettre en avant le rôle de l'entreprise comme élément essentiel de la production des qualités au travail, ce que font les employeurs, et de l'autre, de le nier lorsque la naturalisation de certaines de ces qualités est affirmée ? Ce paradoxe apparaît d'autant plus marqué que certaines des qualités jugées comme naturelles par les employeurs s'apprennent. C'est le cas presque caricatural de la propreté, de la politesse ou de l'élocution, pour reprendre des exemples cités dans les entretiens. On pourrait aussi faire valoir qu'il existe des formations portant sur le comportement de vente, le management, la communication, etc.

Cette contradiction apparaît également dans la tendance des employeurs à faire porter le résultat de l'activité de travail sur le seul jeune en formation : il doit être capable de se sortir par lui-même des épreuves auxquelles il est confronté. De ce point de vue, le jeune en formation n'échappe pas au phénomène de dévolution qui amène l'individu à se sentir, toujours et partout, responsable de ce qui lui arrive et de ce qu'il fait (Martuccelli, 2002). Au-delà de nos entretiens, la figure de "l'apprenti autonome», décrite par Dubrion (2006) dans son étude portant sur les dispositifs d'évaluation des apprentis dans l'enseignement supérieur, en serait une autre illustration. Les critères d'appréciation utilisés pour rendre compte de la qualité du travail réalisé par l'apprenti portent sur sa personnalité. Ils mettent en avant des comportements qui ne concernent pas tant ce que fait le jeune en termes de tâches à exécuter que ce qu'il est, à travers des notions comme l'implication, le sens des responsabilités, l'équilibre personnel ou la résistance physique et psychologique.

L'accent mis par ces employeurs sur la naturalisation des qualités, sur la personnalité de l'individu et sa responsabilité, peut en partie être relié à ce qui définit, dans le commerce et l'hôtellerie-restauration, la professionnalité, c'est-à-dire la capacité à établir un «bon » rapport au client dans un face-à-face sans intermédiaire, où deux subjectivités se rencontrent pour aboutir à un service personnalisé (Hanicotte, 2008). Mais une telle position - et c'est un autre aspect des contradictions des employeurs - revient à minorer la technicité du travail au profit de qualités relationnelles ou comportementales et ainsi le rôle de l'entreprise comme ressource du processus d'apprentissage du jeune et de construction de sa qualité (Lichtenberger, 2007) : l'organisation est simplement considérée comme une donnée à laquelle le jeune doit s'adapter. Plusieurs travaux (Combes, 2002; Grando et Sulzer, 2003) ont toutefois souligné qu'avant de nouer une relation de service (et durant une telle relation), le salarié doit faire preuve de technicité. Si la prise en compte de la relation au client 
est utile, elle ne peut intervenir qu'en second rang. Le salarié doit d'abord maîtriser un certain nombre de ressources (des outils, des matériaux, des procédés) qui relèvent de savoirs et de savoir-faire techniques, de la connaissance de l'entreprise, de son mode de fonctionnement, de sa hiérarchie qui, au final, impliquent véritablement l'organisation. De plus, ces registres de technicité varient d'une activité à l'autre et entretiennent des relations différentes aux diverses modalités d'apprentissage (Grando et Sulzer, 2003). Dans le commerce et l'hôtellerie, même si les registres que les auteurs nomment «technicité industrielle» ou «procédurale» sont présents (maîtrise de logiciels, de procédures de réservation, de technique de présentation, de réassort, d'approche du client...), les employeurs restent très marqués, dans leur approche de la professionnalité, par des registres de technicité qualifiée de "domestique » par Grando et Sulzer, qui relèvent de la personnalité de l'individu. Cette technicité est en quelque sorte «immédiate». Cela amène les employeurs à minorer leur rôle dans le développement de la professionnalité des débutants et contribue à évacuer hors de l'entreprise les questions relatives à la formation de ces qualités. Par opposition, la comptabilité est largement structurée par une technicité procédurale et disciplinaire (un corpus de connaissance et de règles à connaître). Elle requière des apprentissages longs et coûteux, élément largement reconnu par les employeurs.

\section{La reconnaissance professionnelle et le rôle formateur de l'entreprise}

$\mathrm{Au}$-delà de la relation au client, les éléments pointés précédemment, notamment l'accent mis sur l'implication, peuvent aussi s'expliquer par les processus de socialisation et les modèles de réussite sociale en vigueur dans les secteurs du commerce et de l'hôtellerie-restauration. Ces activités sont en effet marquées par la figure idéalisée de l'entrepreneur, c'est-à-dire de l'individu, capable de prendre des risques, responsable de lui-même, ayant le goût de l'effort et qui «s'est construit tout seul», comme l'illustrent les exemples donnés par les employeurs de la grande distribution: "Mon dernier magasin, c'était à Rouville et j'avais mon directeur qui avait commencé comme employé à la boucherie » (Mr B, responsable de rayon, grande distribution), ou encore: "Quand il a commencé, il poussait les caddies, maintenant il est directeur d'un des plus gros magasins de France. » (Mme RP, responsable formation, grande distribution) De même, dans l'hôtellerie-restauration, l'indépendance juridique reste un modèle important de réussite professionnelle : créer, diriger et être le propriétaire de son affaire est une représentation du professionnel qui continue de faire référence dans ce secteur d'activité (Hanicotte, 2008) .

Mais le discours des employeurs n'est sans doute pas non plus indépendant de la reconnaissance professionnelle entendue en un autre sens et qui concerne les questions salariales. D'une part, considérer, comme le font certains employeurs, qu'il existe des qualités qui ne s'apprennent pas et que l'expérience a une fonction de révélation de ces dernières, ne les amène-t-il pas à une moindre reconnaissance de cette expérience et de ces qualités, précisément parce qu'elles sont considérées comme naturelles (Combes, 2002)? L'insistance des employeurs, telle qu'elle ressort des entretiens, à considérer que l'expérience acquise en apprentissage ou en stage ne peut suffire à rendre les futurs salariés directement opérationnels et qu'elle doit être complétée par une expérience professionnelle ultérieure pose le même type de question. D'autre part, en considérant que certaines caractéristiques sont naturelles, les employeurs se désengagent de leur transmission (ou non transmission) et du coût que cela représente, en en reportant la responsabilité (et le coût) sur d'autres, l'école, la famille notamment, comme ils le reconnaissent parfois : «La motivation honnêtement, non, je ne pense pas. On l'a ou on l'a pas, après je pense que c'est... une question d'éducation, et d'état d'esprit, malheureusement... au jour d'aujourd'hui on a beaucoup de jeunes qui sont des enfants rois... » (M. AA, directeur, hôtellerierestauration)

Dans le même temps, les employeurs revendiquent leur rôle de formateur et y semblent très attachés, d'où les contradictions relevées précédemment dans la grande distribution et l'hôtellerie-restauration.

\footnotetext{
${ }^{5}$ Aujourd'hui encore, les hôtels indépendants représentent $85 \%$ du parc hôtelier, et plus d'un tiers des établissements (restauration et hôtellerie confondues) n'a pas de salarié.
} 
Comment comprendre cet attachement? La question est délicate. Il faut en effet tenir compte des stratégies multiples d'utilisation du dispositif de l'apprentissage par les employeurs (Lhotel et Monaco, 1993 ; Léné, 2000). Quelques réponses peuvent être esquissées. L'attachement des entreprises à leur rôle de formateur peut d'abord tenir aux avantages financiers liés à l'accueil des jeunes en formation. Il peut ensuite être lié à leur conviction qu'une partie des qualités, des connaissances nécessaires au travail ne s'apprend véritablement qu'en situation de travail. On l'a déjà signalé. La peur de manquer de personnel sur certains métiers particuliers, parfois très pénibles, est aussi mise en avant dans les entretiens. Enfin, la période de la scolarité passée en entreprise peut être vue par les employeurs davantage comme un moyen d'observer et de repérer, parmi les jeunes, ceux qui font la preuve de qualités attendues, que d'accueillir des débutants pour les former (Vincens, 2001).

\section{Le rôle instable de l'entreprise dans le système de formation}

Ces différents constats questionnent l'organisation des modes de formation en alternance. D'abord, la période de la scolarité passée en entreprise peut aussi avoir un rôle de tri ou de repérage des jeunes les plus conformes aux attentes des employeurs, c'est-à-dire de sélection, comme on l'a indiqué. Or l'apprentissage est déjà un mode de formation sélectif : trouver une entreprise, condition nécessaire pour se former dans cette filière, est difficile (Moreau, 2002). De nombreux candidats sont contraints d'abandonner faute d'avoir pu trouver une entreprise d'accueil. Le tri opéré par l'apprentissage semble donc se poursuivre au-delà de l'entrée en entreprise. Nos résultats tendent à montrer qu'il est à l'œuvre pendant tout le parcours du jeune et structure l'attitude générale de l'entreprise vis-à-vis du jeune.

Ensuite, le rôle de l'entreprise dans le processus de formation n'est pas encore stabilisé du point de vue institutionnel (Léné, 2002). En France, malgré les progrès de l'alternance, n'émerge pas un système d'intégration réglementée à l'allemande où la législation précise dans les moindres détails les conditions d'emploi des apprentis et leur formation (la présence d'un Meister - maître d'apprentissage - dans la firme est par exemple obligatoire ; celui-ci doit avoir reçu une formation de formateur). Cette question nous semble particulièrement vive dans les métiers de services où la composante relationnelle de la compétence ainsi que l'implication subjective des jeunes sont particulièrement mises en avant. S'il est largement reconnu que l'aspect technique du savoirfaire s'acquiert en partie par la pratique et que son acquisition nécessite un accompagnement et une organisation de l'activité spécifique pour le jeune débutant, les choses sont beaucoup moins évidentes pour les composantes relationnelles ou comportementales de l'activité de travail. La question de la formation en alternance dans les activités en émergence, ou qui ne sont pas encore stabilisées du point de vue de la professionnalité des métiers qui y sont exercés, apparaît ainsi selon nous comme un enjeu majeur. C'est par exemple le cas de l'hôtellerie. Catherine Agulhon (2003) rappelle ainsi qu'il s'agit d'un secteur dont les caractéristiques professionnelles le distinguent nettement de secteurs organisés comme la grande industrie, les banques ou les assurances. Il n'a signé sa convention collective nationale qu'en avril 1997, ce qui ne garantit pas son application. Ce secteur ne se caractérise ni par son respect de la législation du travail, ni par sa capacité à construire ses propres normes.

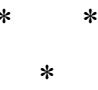

L'objectif de cet article était de s'interroger sur le caractère formateur des périodes de la scolarité passée en entreprises, qui occupent une place importante dans les filières de formation professionnelle. L'analyse a été menée d'un point de vue empirique à partir d'entretiens réalisés auprès d'employeurs du secteur des services, en France. De l'exploitation de ces entretiens, il apparaît que la période en entreprise n'est pas mécaniquement formatrice. Les employeurs de la grande distribution et de l'hôtellerie-restauration insistent en effet sur l'implication et la responsabilisation des jeunes débutants. Ils considèrent également qu'une partie des qualités nécessaires au travail ne s'apprend pas, comme par exemple le sens du commerce, le contact avec le client, les qualités relationnelles... La période de la scolarité passée en entreprise apparait alors comme un moyen de sélectionner les candidats conformes aux attentes des 
employeurs. Même lorsqu'une finalité formatrice est recherchée, la production de connaissances, de formation, ne va pas de soi. Elle nécessite des conditions auxquelles une attention particulière nous semble devoir être accordée.

Ces différents éléments relativisent le lien mécanique souvent établi entre expérience, temps de présence en entreprise et connaissance. Plus largement, ils amènent à reconsidérer la capacité des employeurs à assumer leur rôle dans la formation des jeunes. Ils soulignent que ce rôle est influencé par la représentation qu'ils se font de la portée formatrice du travail : si l'entreprise peut être effectivement un lieu de formation, encore faut-il que les employeurs acceptent l'idée que les débutants sont en position d'apprentissage et que l'organisation contribue à cet apprentissage.

\section{Bibliographie}

Agulhon C. (2002), « Les incidences socio-économiques de l'alternance», in G. Moreau (Ed.), Les patrons, l'État et la formation des jeunes, septembre, Paris, La Dispute, pp. 141-152.

Agulhon C. (2003), «Diplômes-expériences : complémentarité ou concurrence. Le cas de l'hôtellerie ", L'orientation scolaire et professionnelle, vol. $32, \mathrm{n}^{\circ} 1$, pp. 53-73.

Argyris C., Schon D.A. (1978), Organizational Learning, Reading, MA: Addison-Wesley.

Arrow K. (1962), «The economic implications of learning-by-doing », Review of Economic Studies, june, pp. 155-73.

Ashton D.N. (2004), « The impact of organisational structure and practices on learning in the workplace », International Journal of Training \& Development, vol. 8, $\mathrm{n}^{\circ} 1$, pp. 43-53.

Bailly F. (2004), « Contenus de formation et activités de travail : les jugements des recruteurs ", in Chatel E., Capacités d'agir et formation scolaire. Une étude des baccalauréats professionnels et technologiques tertiaires, Rapport final pour le Commissariat Général du Plan en réponse à l'appel d'offre «L'évaluation du système d'éducation et de formation ».
Bailly F. (2008), "The role of employers' beliefs in the evaluation of educational output", The Journal of Socio-Economics, vol. 37, n 3, pp. 959-968.

Bailly F., Chatel E., Léné A. (2008). Expérience et formation par la pratique. Évaluation d'employeurs et de salariés du tertiaire, Rapport de recherche pour l'ACI «Éducation et formation: contextes et effets », mars.

Baker H.E., Feldman D.C. (1990), "Strategies of organizational socialization and their impact on newcomer adjustmen" ", Journal of Managerial Issues, vol. 2. pp. 198-212.

Balsan D., Hanchane S., Werquin P. (1996), «Mobilité professionnelle initiale: éducation et expérience sur le marché du travail. Un modèle Probit à effet aléatoire ", Économie et Statistique, $\mathrm{n}^{\circ} 299$.

Becker G.S. (1964), Human Capital, a theorical and empirical analysis, with special reference to education, $2^{\mathrm{e}}$ edition, New York, Columbia University Press.

Béduwé C., Espinasse J.-M., Tahar G. (2000), «Inexpérience professionnelle et accès des jeunes à l'emploi », Note du LIRHE, $\mathrm{n}^{\circ} 313$, juin.

Bellier S. (1998), Le savoir-être dans l'entreprise: utilité en gestion des ressources humaines, Vuibert, Paris. 
Bewley T. (2002), "Interviews as a valid empirical tool in economics", The Journal of Socio-Economics, vol. 31 .

Blinder A.S. (1990), "Learning by Asking Those Who Are Doing", Eastern Economic Journal, vol. 16 .

Blinder A.S., Weiss Y. (1976), "Human Capital and Labor Supply: a synthesis", Journal of Political Economy, vol. 84, n 3, June, pp. 449-72.

Bonnal L., Clément D., Mendes S. (2005), « L'accès au premier emploi au cours des années 1990 : le cas des apprentis et des lycéens », Économie et Statistique, $\mathrm{n}^{\circ} 378-379$.

Bonnal L., Fleury L., Rochard M.-B. (1999), «L'insertion professionnelle des apprentis et des lycéens professionnels: des emplois proches des formations suivies », Économie et Statistique, $323(3)$.

Chatel E. (2007), "Les débuts dans la vie professionnelle : une analyse inspirée de la philosophie de J. Dewey ", Communication au Colloque « Savoirs et expériences », Amiens, mars.

Combes M.-C. (1996), L'alternance: enjeux et débats, DARES, La Documentation française, Paris.

Combes M.-C. (2002), « La compétence relationnelle : une question d'organisation », Travail et Emploi, $\mathrm{n}^{\circ} 92$.

Dubernet A.-C. (1996), « La sélection des qualités dans l'embauche. Une mise en scène de la valeur sociale », Formation Emploi, n ${ }^{\circ}$ 54, avril-juin, pp. 3-14.

Dubrion B. (2006), «Qu'est-ce qu'un “bon apprenti” du supérieur? », Formation Emploi, n 96, octobredécembre, pp. 93-105.

Eymard-Duvernay F., Marchal E. (1997), Façons de recruter : le jugement des compétences sur le marché du travail, Métailié, Paris.

Favereau O. (1986), « Evolution récente des modèles et des représentations théoriques du fonctionnement du marché du travail», Problèmes Economiques, pp. 3-14.
Flueckiger G.E. (1976), "Specialisation, learning by doing and the optimal amount of learning", Economic Inquiry, September, pp. 389-409.

Foray D. (2001), L'économie de la connaissance, Éditions La Découverte, coll. « repères » $n^{\circ} 302$.

Gadrey J. (2003), Socio-économie des services, La Découverte, coll. « Repères ».

Gadrey N., Jany-Catrice F., Pernod-Lemaître M. (2003), Les enjeux de la qualification des employés. Conditions de travail et compétences des "non qualifiés », Rapport pour le ministère de l'Emploi et de la Solidarité, DARES.

Grando J.-M., Sulzer E. (2003), « Professionnalisation des activités de service et registres de technicité », in Menger P.-M. (Ed.), Les professions et leurs sociologies. Modèles théoriques, catégorisation, évolutions, Éditions de la Maison des Sciences de l'Homme, Paris.

Grasser B. (1996), "Organization and work situations: which sources for the learning process?", Labour, vol. 10, n 1, pp. 63-92.

Grasser B., Rose J. (2000), «L'expérience professionnelle, son acquisition et ses liens à la formation 》, Formation Emploi, $\mathrm{n}^{\circ} 71$, juilletseptembre, pp. 5-19.

Guile D., Griffiths T. (2001), "Learning Through Work Experience", Journal of Education \& Work, vol. $14, \mathrm{n}^{\circ} 1$, pp. 113-131.

Hanicotte P. (2008), «Les modèles de réussite sociale comme révélateurs des logiques de professionnalisation. Le cas de la figure de l'entrepreneur dans l'hôtellerie de chaîne", séminaire SERAS, Clersé, université de Lille 1, 17 janvier.

Koike K. (2002), "Intellectual Skills and Competitive Strength: is a radical change necessary?", Journal of Education and Work, vol. 14, n 4, pp. 390-408.

Lhotel H., Monaco A. (1993) «Regards croisés sur l'apprentissage et les contrats de qualification », Formation Emploi, ${ }^{\circ}$ 42, avril-juin, pp. 33-45. 
Lemelin C. (1998), L'économiste et l'éducation, Presse Universitaire du Québec.

Léné A. (2000), « Le fragile équilibre de la formation en alternance: un point de vue économique », Formation Emploi, n ${ }^{\circ}$ 72, pp. 15-33.

Léné A. (2002), Formation, compétences et adaptabilité. L'alternance en débat, Éditions L'Harmattan, Paris.

Levy-Garboua L. (1994), «Formation sur le tas et rendements de l'expérience : un modèle de diffusion du savoir », Économie et Prévision, n ${ }^{\circ} 116$, pp. 79-88.

Lichtenberger Y. (2007), «Organisation, travail et compétence » in Campinos-Dubernet M., Combes M.-C., Redor D. (Eds.), Les mutations des industries et des services, Octarès, Toulouse.

Light A. (2001), "In-school Work Experience and the Returns to Schooling", Journal of Labor Economics, vol. $19, \mathrm{n}^{\circ}$ pp. $65-93$.

March J.G., Simon H.A. (1958), Organizations, Wiley, New York.

Malglaive G. (1996), «Apprentissage, une autre formation pour d'autres ingénieurs », Formation Emploi, ${ }^{\circ}$ 53, janvier-mars, pp. 85-99.

Mallet L., Vernieres M. (1981), «L'expérience professionnelle dans les modes de gestion de la main-d'œuvre », Travail et Emploi, $\mathrm{n}^{\circ}$ 9, juilletseptembre, pp. 65-74.

Martuccelli D. (2002), Grammaires de l'individu, Paris, Gallimard.

Merle V. (1994), « Objectifs et organisation pédagogique de l'alternance ", in OCDE et CEREQ (Eds.), Les formations en alternance : quel avenir ?, Paris, pp. 29-40.

Mincer J. (1958), "Investment in human capital and personal income distribution", Journal of Political Economy, vol. 4.

Molitor C. J., Leigh D. E. (2005), "In-school work experience and the returns to two-year and four-year colleges", Economics of Education Review, 24, pp. 459-468.

Moreau G. (2002), Les patrons, l'État et la formation des jeunes, septembre, Paris, La Dispute.

Orléan A. (2002), «Le tournant cognitif en économie », Revue d'économie politique, 112 (5), pp. 717-738.

Rosen S. (1972a), "Learning by experience as joint production", Quarterly Journal of Economics, vol. $86, \mathrm{n}^{\circ} 3$.

Rosen S. (1972b), "Learning and Experience in the Labor Market", The Journal of Human Resources, vol. $7, \mathrm{n}^{\circ} 3$.

Salognon M. (2006), « Modes de jugement des entreprises et insertion des chômeurs de longue durée. Observation de la méthode IOD », in Dang A.-T., Outin J.-L. et Zajdela H. (Eds.), Travailler pour être intégré? Mutations des relations entre emploi et protection sociale, Éditions du CNRS, septembre.

Savoyant A. (1996), "Une approche cognitive de l'alternance », Bref-Cereq, n ${ }^{\circ} 118$, mars.

Spence M. (1973), “Job Market Signaling”, Quarterly Journal of Economics.

Stankiewicz F. (2002), «Productivité ou "valorité" du salarié ? Contribution à l'analyse du travail», Travail et Emploi, $\mathrm{n}^{\circ} 91$.

Stiglitz J.E. (1987), "Learning to learn, localized learning and technological progress", in Dasgupta P., Stoneman P. (Eds), Economic policy and technological performance, Cambridge U.P., pp. 125-53.

Toupin L. (1991), «Formation expérientielle et transfert de connaissances ", in Courtois B., Pineau G. (Eds.), La formation expérientielle des adultes, La Documentation française, Paris.

Ughetto P., Besucco N., Tallard M., Du Tertre C. (2002), «La relation de service : Une tension vers un nouveau modèle de travail », Revue de l'Ires, $n^{\circ} 39$.

Verdier E. (2001), «La France a-t-elle changé de régime d'éducation et de formation? » Formation Emploi, $\mathrm{n}^{\circ}$ 76, pp. 11-34. 
Vergnaud G. (1991), «Pourquoi la psychologie cognitive ? », La Pensée, n 282, juillet-août, pp. 9-19.
Vincens J. (2001), « Définir l'expérience professionnelle », Travail et Emploi, vol. 85.

\section{Résumé}

\section{La portée formatrice de l'expérience : entretiens auprès d'employeurs du secteur des services}

Franck Bailly, Alexandre Léné et Marie-Hélène Toutin

Les périodes de la scolarité passées en entreprises occupent une place importante dans les dispositifs de formation professionnelle en France. Presque naturellement, une portée formatrice leur est attribuée. Cet article interroge ce lien mécanique entre temps en entreprise, expérience et connaissances. L'exploitation d'entretiens réalisés auprès d'employeurs du secteur des services montre que, selon ces derniers, la période en entreprise peut constituer un moment formateur mais également révéler des qualités déjà possédées par le salarié. Après avoir interrogé les représentations des employeurs, les auteurs tentent de caractériser les éléments significatifs dans la production d'expérience. Sur cette base, ils questionnent de façon critique le rôle des périodes passées en entreprise des filières de formation fondées sur l'alternance.

\section{Mots clés}

Formation en alternance, apprentissage, expérience professionnelle, organisation qualifiante, secteur tertiaire

Journal of Economic Literature: M 53 